\title{
Challenges in Imaging Single Atoms Adsorbed or Embedded on Surfaces
}

\author{
Jingyue (Jimmy) Liu* and Lawrence F. Allard** \\ * Center for Nanoscience, Department of Physics and Astronomy, Department of Chemistry and \\ Biochemistry, University of Missouri-St. Louis, St. Louis, MO 63121 (liuj@umsl.edu)** Materials \\ Science \& Technology Div., Oak Ridge National Laboratory, Oak Ridge, TN 37831
}

Visualization of single atoms of heavy elements supported on light-element substrates was achieved in the early days of scanning transmission electron microscopy (STEM) [1]. Applications of the high-angle annular dark-field (HAADF) technique to imaging single atoms and small clusters either dispersed on catalyst supports or doped into semiconductor materials have been reported [2-3]. In crtain cases, spectroscopic identification and imaging of individual atoms in bulk materials or in encapsulated state have been realized [4-5]. The use of low accelerating voltages mitigates the effects of electron beam irradiation, and individual atoms of even light elements have been identified and imaged [6-7]. For many practical applications, especially for imaging and identification of surface adsorbed atoms (adatoms) or ad layers in practical heterogeneous catalysts, the electron beam- induced effects in aberration-corrected electron microscopes still pose a formidable challenge.

Under the intense electron beam irradiation in aberration-corrected STEM, single atom s usually move around and do not maintain their spatial relationship with the substrate. The most typical example is Au atoms or clusters on an amorphous carbon film [8]. Figures 1a and $1 \mathrm{~b}$ show two images of $\mathrm{Au}$ nanoparticles supported on amorphous carbon; the two images were recorded consecutively. Individual $\mathrm{Au}$ atom s moved around on the substrate, and the small Au particle also rapidly changed its shape under electron beam irradiation. Some single $\mathrm{Au}$ atoms were emitted from the Au nanoparticles while other $\mathrm{Au}$ atoms joined the Au nanoparticles. This behavior precludes the ability to obtain unambiguous information on the intact state of the $\mathrm{Au} /$ carbon sample.

When the adatoms form a strong bond with the substrate, however, the adatoms or adlayers can be stable for a reasonable amount of time to allow extraction of useful information about their relationship with the support materials. This usually occurs when the substrate is a crystalline material and when the adatoms or adlayers form a specific structural relationship with the substrate surface. Figure 2a shows an example of such a relationship; individual $\mathrm{Pt}$ atoms were adsorbed onto a $\mathrm{SiC}$ crystal and in some regions formed an ordered two-dimensional adlayer. Figure $2 \mathrm{~b}$ shows individual $\mathrm{Pd}$ atom $\mathrm{s}$ adsorbed onto $\mathrm{ZnO}$ nanowires, providing information on the structural relationship between the $\mathrm{Pd}$ atom $\mathrm{s}$ and the $\mathrm{ZnO}$ surface sites; the presence of $\mathrm{s}$ ingle $\mathrm{Pd}$ atoms significantly modified the performance of the $\mathrm{Pd} / \mathrm{ZnO}$ nanocatalyst for the methanol steam reforming reaction. Figure 3 shows another example of the power of HAADF imaging of surface adsorbed atoms on relatively thick, but practical supports. In this case, the individual $\mathrm{Pd}$ atoms were stable during the observation, and they appeared to be embedded onto the (11-20) surface of the thick ZnO nanobelt (D FT calculations confirmed the possibility of this configuration). Imaging strategies to visualize surface adatoms and adlayers in practical nanocatalysts will be discussed [9].

\section{References}

[1] A.V. Crewe, J. Wall and J. Langmore, Science, 168 (1970) 1338.

[2] P.D. Nellist and S.J. Pennycook, Science, 274 (1996) 413.

[3] P.M. Voyles et al., Nature, 416 (2002) 826.

[4] K. Suenaga et al, Science, 290 (2000) 2280.

[5] M. Varela et al., Phys. Rev. Lett., 92 (2004) 095502. 
[7] O.L. Krivanek et al., Nature, 464 (2010) 571.

[8] P.E. Batson, N. Dellby and O.L. Krivanek, Nature, 418 (2002) 617.

[9] This research was supported by UM-St. Louis; microscopy research at the Oak Ridge National Laboratory's High Temperature Materials Laboratory was sponsored by the U. S. Department of Energy, Office of Energy Efficiency and Renewable Energy, Vehicle Technologies Program. 